\title{
Proposal for a gold standard for cosmetic evaluation after breast conserving therapy: Results from the St George and Wollongong Breast Boost trial
} \author{
Peter Graham, ${ }^{1}$ Alison Szwajcer ${ }^{1}$ and Eric $\mathrm{Hau}^{4}$ \\ 1 St George Cancer Care Centre, Kogarah, New South Wales, Australia \\ 2 Faculty of Engineering, University of Porto, Porto, Portugal \\ 3 Breast Cancer Unit, Champalimaud Cancer Centre, Lisbon, Portugal \\ 4 Crown Princess Mary Westmead Cancer Care Centre, Westmead, New South Wales, Australia
}

Roya Merie, ${ }^{1}$ (D) Lois Browne, ${ }^{1}$ Jaime S Cardoso, ${ }^{2}$ Maria J Cardoso, ${ }^{3}$ Yaw Chin, ${ }^{1}$ Catherine Clark, ${ }^{1}$

\begin{abstract}
R Merie MD; L Browne PhD; JS Cardoso PhD; MJ Cardoso PhD; Y Chin FRANZCR; C Clark FRANZCR; P Graham FRANZCR; A Szwajcer MNSC; E Hau FRANZCR.

\section{Correspondence}

Dr Roya Merie, Liverpool Cancer Therapy Centre, Liverpool, NSW 2170, Australia. Email: Roya.Merie@sswahs.nsw.gov.au
\end{abstract}

Conflict of interest: None.

Submitted 12 March 2017; accepted 2 July 2017.

doi:10.1111/1754-9485.12645

\begin{abstract}
Introduction: Breast cosmesis is an important endpoint of breast conserving therapy $(B C T)$, but a gold standard method of its evaluation is not yet established. The St. George and Wollongong Randomised Breast Boost trial used five different methods of cosmetic assessment, including both subjective and objective, to comprehensively evaluate the cosmetic outcome of the trial patients. This current study analyses the level of concordance between these methods in an attempt to determine a possible standard in the evaluation of breast cosmesis.
\end{abstract}

Methods: Patients attending follow-up clinic reviews at 5 years post breast radiotherapy were evaluated. Patients completed a cosmesis and functional assessment questionnaire, assessing clinicians completed an EORTC (European Organization for Research and Treatment of Cancer) cosmetic rating questionnaire and photographs were obtained. The photographs were later assessed by a panel of five experts, as well as analysed using the objective pBRA (relative Breast Retraction Assessment) and the BCCT.core (Breast Cancer Conservative Treatment.cosmetic results) computer software. Scores were dichotomised to excellent/good and fair/poor. Pairwise comparisons between all methods, except pBRA, were carried out using overall agreement calculations and kappa scores. pBRA scores were compared on a continuous scale with each of the other dichotomised scores obtained by the other four methods.

Results: Of 513 St George patients alive at 5 years, 385 (75\%) attended St George for follow-up and consented to photography. Results showed that assessment by physicians in clinic and patient self-assessment were more favourable regarding overall cosmetic outcome than evaluation of photographs by the panel or the BCCT.core software. Excellent/good scores by clinician-live and patient self-assessments were $93 \%$ and $94 \%$ respectively (agreement $89 \%$ ), as compared to $75 \%$ and $74 \%$ only by BCCT.core and panel assessments respectively (agreement 83\%, kappa 0.57). For the pBRA measurements, there was a statistically significant difference $(P<0.001)$ between scores for excellent/good versus fair/poor cosmesis by all four methods. The range of median pBRA measurements for fair/poor scores was 13.4-14.8 and for excellent/good scores was 8.0-9.4.

Conclusion: Incorporating both BCCT.core assessment and patient self-assessment could potentially provide the basis of a gold standard method of breast cosmetic evaluation. BCCT.core represents an easy, time efficient, reproducible, cost effective and reliable method, however, it lacks the functional 
and psychosocial elements of cosmesis that only patient self-reported outcomes can provide.

Key words: BCCT.core; breast conserving therapy; breast cosmesis; breast radiotherapy; cosmetic evaluation.

\section{Introduction}

With the proven equivalent oncological outcomes between mastectomy and breast conserving therapy $(\mathrm{BCT}),{ }^{1-5}$ a good cosmetic result has become an increasingly important endpoint of BCT. To date, however, an ideal and standardised method of assessing cosmesis after BCT is not yet established. ${ }^{6}$ Across the multiple large randomised trials that evaluated effect of different radiotherapy techniques and fractionation schedules on breast cosmesis as one of the endpoints, there has been a clear lack of consistency in the evaluation techniques. For example, in the UK START trial A, photographic comparison with baseline, patient self-reported assessments, and physician assessments were used. ${ }^{7}$ The published long-term results of the Canadian hypofractionated radiotherapy for breast cancer, has only reported global cosmetic outcome as assessed by a clinical-trials nurse using the EORTC scale. ${ }^{8}$ In the UK randomised trial of IMRT use in early breast cancer, superior cosmetic outcomes were reported based on photographic as well as clinical assessments using a validated three-point scale. ${ }^{9}$

Having a standardised method of assessing cosmesis not only would allow comparison between the results of the different published trials, but would also enable the identification of the different surgical and radiotherapyrelated variables that will affect the final aesthetic result. ${ }^{10}$ And with the upcoming new oncoplastic interventions and the introduction of partial breast irradiation techniques, there will be even more demand for a standardised evaluation of aesthetic results to help tailor the spectrum of techniques available to individual cases and improve them. ${ }^{11}$ These new treatment modalities should be evaluated using reliable, time efficient and reproducible tools. ${ }^{12}$

Various scales of assessments have been used to assess cosmesis. Harris et al. ${ }^{13}$ were the first to describe a qualitative scoring system (Harvard scale), classifying the cosmetic outcome into one of four categories (excellent, good, fair or poor) by comparing the treated breast with the untreated one. Pezner et al., on the other hand, concluded that this scoring system can rarely result in observer consensus, whereas dichotomised grading into good versus poor cosmetic outcome will substantially improve the observer consensus. ${ }^{14,15}$

The various reported methods of assessing cosmesis after BCT can be broadly divided into subjective and objective methods. ${ }^{16-19}$ For both groups of methods, evaluation can be carried out directly on patients or by means of photographs. The St. George and Wollongong (SGW) randomised breast boost study that aimed at evaluating the impact of a radiotherapy boost on breast cosmesis, utilised subjective panel assessment, patient and clinician-live assessments, objective relative Breast Retraction Assessment ( $\mathrm{pBRA}$ ) tool and the BCCT.core 2.0 (the Breast Cancer Conservative Treatment.cosmetic results version 2.0 ) software for a comprehensive evaluation. ${ }^{20}$

This current study focuses on assessing the degree of agreement between these methods and aims to postulate a gold standard for breast cosmetic evaluation.

\section{Methods}

Patients of the St. George and Wollongong (SGW) randomised breast boost trial ${ }^{20}$ (NCT 00138814) who attended the follow-up clinic review at 5 years post their breast radiotherapy have been evaluated. The evaluation consisted of: (i) Cosmesis and functional assessment questionnaire completed by the patients themselves with the overall cosmesis scored as 'normal', 'excellent', 'good', 'fair' or 'poor', (ii) Live assessment by the treating clinician who scored the overall cosmesis as 'excellent', 'good', 'fair' or 'poor', (iii) Frontal photos in the akimbo (hands on hips) position, and these were later assessed objectively by PBRA and BCCT.core software, and subjectively by a five-person panel scoring the overall cosmesis again as 'excellent', 'good', 'fair' or 'poor'. The panel consisted of two radiation oncologists, one breast surgeon, one breast clinical nurse consultant and one radiotherapy clinical nurse specialist (four women, one man), all with experience in the treatment of breast cancers.

The BRA measures the degree of breast retraction compared with the untreated breast ${ }^{21}$ and PBRA is defined as (BRA/reference length) $\times 100$. The BCCT.core software was developed by Jaime and Maria-João Cardoso in 2007, which quickly and automatically integrates the indices correlated with overall cosmesis such as asymmetry, colour and surgical scar visibility into an overall global cosmetic score. ${ }^{22,23}$

We analysed the scores obtained by the five different methods outlined above. The scores were dichotomised to excellent/good (normal/excellent/good in case of patients' self-assessment) and fair/poor. Pairwise comparison between the scores was then carried out using overall agreement and kappa scores. pBRA scores on a continuous scale were compared to each of the dichotomised scores by Wilcoxon rank-sum test. Data were 
analysed using STATA version 11 (StataCorp, College State, TX, USA).

\section{Results}

Of the 688 patients randomised in the SGW breast boost trial, 513 were alive at 5 years, and of these, 385 (75\%) presented for follow-up at St. George Hospital and consented to photography. Assessment of the images by the panel and BCCT.core provided scores for all the 385 patients, whereas pBRA scores were available for 383 patients. Scores obtained by clinician assessment in clinic and patient self-assessment were available for 374 and 362 patients respectively (Fig. 1).

Categorical as well as dichotomised scores for all methods of assessment, except pBRA, are summarised in Table 1.

As shown in Table 1, assessments by 'clinician' and 'patient' showed few scoring either fair or poor. These assessments were therefore less critical of overall cosmetic result than assessments using patient images. Excellent/good assessments of patients live were $93 \%$ by clinician and $94 \%$ by patient, whereas excellent/good assessments of patient images were $75 \%$ by BCCT.core and $74 \%$ by the panel.

Comparisons between the four categorical scales showed the highest percentage agreement of $89 \%$ was between the two scoring methods of patient live
(Table 2). The second highest percentage agreement was $83 \%$ between the two image scores by BCCT.core and the Panel. The other four comparisons between a live patient score and an image score gave agreements of $75 \%$ or $76 \%$.

Kappa scores are appropriate to test whether agreement exceeds chance and all scores indicate this, however, the scores are generally low (Table 2). The only kappa above 0.25 was BCCT versus Panel with a kappa of 0.57.

For all four categorical scales, there was an increase in the median PBRA measurement for scores grading from excellent to good, good to fair and fair to poor (Table 3). There was a statistically significant difference between dichotomised scores of excellent/good versus fair/poor for all four scales. The range of median pBRA measurements for fair/poor scores was 13.4-14.8 and for excellent/good scores was 8.0-9.4. There was a greater difference between median values of the image-generated score of pBRA with the image scores of BCCT.core and Panel (6.8, 5.5 respectively) compared to live scores (5.0 for clinician and 4.0 for patient). The largest difference in median pBRA score was the other computer generated score BCCT.core.

\section{Discussion}

Our results indicate that direct assessment of cosmetic outcome by the clinician in clinic or by the patients

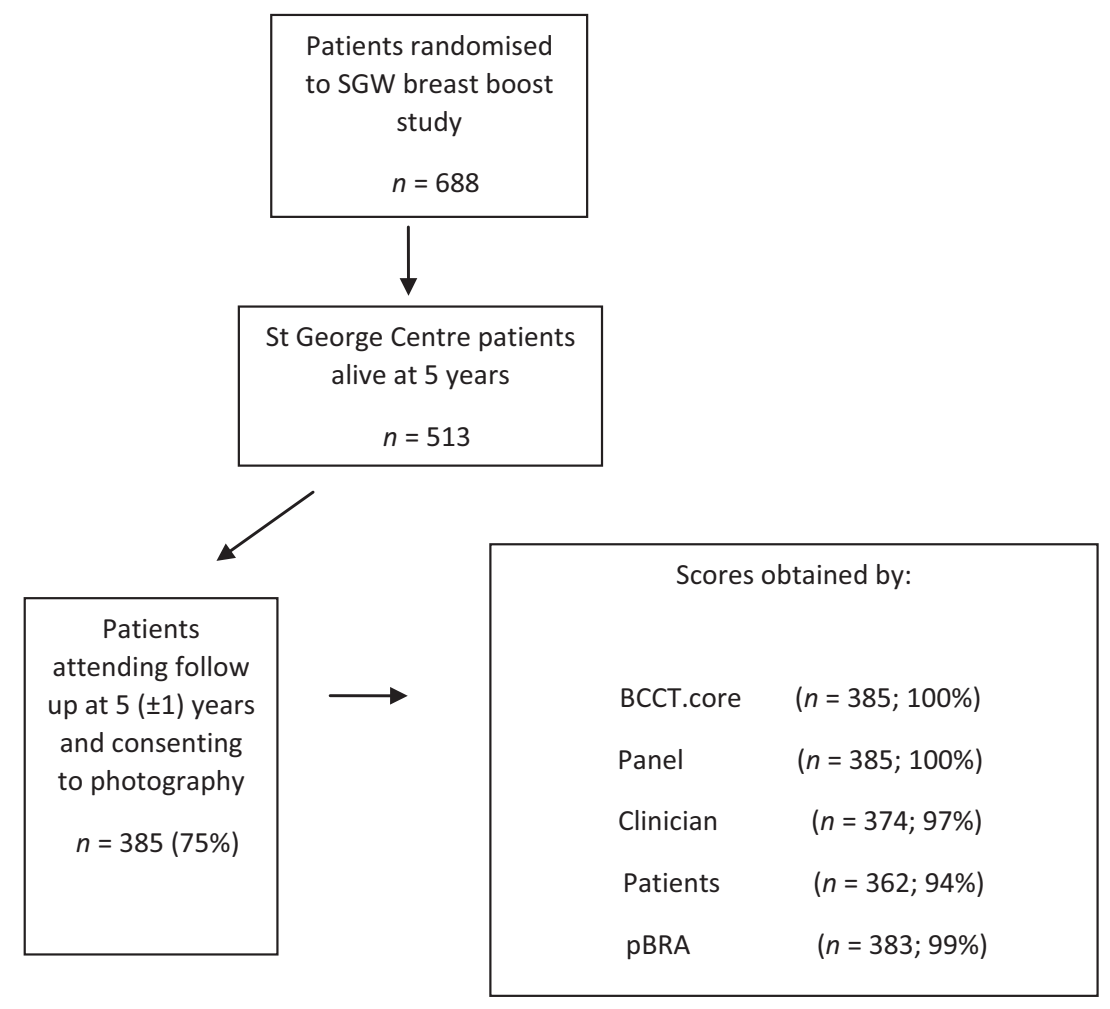

Fig. 1. Study flow chart. 
Table 1. Categorical and dichotomized scores of the cosmetic assessment methods used in the SGW breast boost study

\begin{tabular}{|c|c|c|c|c|c|c|c|c|}
\hline \multirow[t]{3}{*}{ Assessed by } & \multicolumn{4}{|c|}{ Score, $n(\%)$} & \multicolumn{4}{|c|}{ Dichotomised Score } \\
\hline & \multirow[t]{2}{*}{$\begin{array}{l}\text { Excellent } \\
\text { or normal }\end{array}$} & \multirow[t]{2}{*}{ Good } & \multirow[t]{2}{*}{ Fair } & \multirow[t]{2}{*}{ Poor } & \multicolumn{2}{|c|}{$\begin{array}{c}\text { Excellent, good or } \\
\text { normal }\end{array}$} & \multicolumn{2}{|c|}{ Fair or poor } \\
\hline & & & & & $n(\%)$ & $\begin{array}{c}95 \% \mathrm{Cl} \\
\%\end{array}$ & $n(\%)$ & $\begin{array}{c}95 \% \mathrm{Cl} \\
\%\end{array}$ \\
\hline \multicolumn{9}{|l|}{ Image scoring } \\
\hline BCCT.core & $127(33)$ & $160(42)$ & $82(21)$ & $16(4)$ & $287(75)$ & $70-79$ & $98(25)$ & $21-30$ \\
\hline Panel & $130(34)$ & $153(40)$ & $89(23)$ & $13(3)$ & $283(74)$ & $69-78$ & $102(26)$ & $22-31$ \\
\hline \multicolumn{9}{|c|}{ Patient 'live' scoring $\dagger$} \\
\hline Clinician & $220(59)$ & $126(33)$ & $22(6)$ & $6(2)$ & 346 (93) & $89-95$ & $28(7)$ & $5-11$ \\
\hline Patient & $202(56)$ & $138(38)$ & $19(5)$ & $3(1)$ & 340 (94) & $91-96$ & $22(6)$ & $4-9$ \\
\hline
\end{tabular}

$\mathrm{Cl}$, confidence interval.

$\dagger$ Some missing scores.

Table 2. Pairwise comparisons of four dichotomised scores

\begin{tabular}{|c|c|c|c|c|}
\hline & \multirow{2}{*}{$\begin{array}{l}\text { Agreement } \\
\text { overall, \% }\end{array}$} & \multicolumn{2}{|c|}{ Agreement, \% } & \multirow{2}{*}{$\begin{array}{l}\text { Kappa score } \\
(95 \% \mathrm{Cl} \%)\end{array}$} \\
\hline & & $\begin{array}{l}\text { Excellent } \\
\text { or good }\end{array}$ & $\begin{array}{l}\text { Fair } \\
\text { or poor }\end{array}$ & \\
\hline \multicolumn{5}{|l|}{ Image vs image } \\
\hline BCCT vs Panel & 83 & 65 & 18 & $0.57(0.47-0.67)$ \\
\hline \multicolumn{5}{|l|}{$\begin{array}{l}\text { Patient live vs } \\
\text { patient live }\end{array}$} \\
\hline Clinician vs patient & 89 & 88 & 1 & $0.15(0.05-0.25)$ \\
\hline \multicolumn{5}{|l|}{ Image vs patient live } \\
\hline BCCT vs clinician & 76 & 71 & 5 & $0.18(0.10-0.26)$ \\
\hline BCCT vs patient & 75 & 72 & 3 & $0.12(0.05-0.20)$ \\
\hline Panel vs clinician & 76 & 71 & 5 & $0.21(0.13-0.28)$ \\
\hline Panel vs patient & 75 & 71 & 4 & $0.14(0.07-0.22)$ \\
\hline
\end{tabular}

vs, versus.

themselves is less critical than assessment obtained by means of photographs. Most authors in the literature found that patients tend to score cosmesis more favourably than physicians. ${ }^{21,24-27}$ This is consistent with our findings if we compare panel evaluation (74\% for excellent/good scores) with patient's self-assessment ( $94 \%$ scores normal/excellent/good outcomes). Scores obtained by direct clinician assessment versus patient self-assessment on the other hand, were closely similar (93\% and $94 \%$ respectively). This could possibly be explained by the positive impact of the direct interaction between the physician and the patient who is pleased with the cosmetic outcome. This impact is absent when assessment is done merely on photographs.

Agreement between the different cosmetic evaluation methods in this study was tested using kappa scores. All values were above zero indicating that the agreement exceeded chance. These values were, however, generally low (Table 2). The only kappa above 0.25 was BCCT versus Panel with a kappa of 0.57. For this comparison, there were reasonable numbers scoring as fair/poor $(n=98,102)$ and percentages were also similar $(25 \%$, $26 \%$ ). For all other comparisons, one of the scores has a low prevalence of fair/poor ( $6 \%$ or $7 \%$ ). The kappa value of 0.15 for clinician versus patient would seem surprisingly low given $89 \%$ overall agreement. This is due to the low prevalence of fair/poor scoring for both scales resulting in an agreement of $1 \%$ for fair/poor score. Kappa scores are affected by both prevalence and bias between scores. ${ }^{28}$ All four kappa scores comparing an image score with a live patient score are low (0.12-0.21) as they are comparing scores of different prevalence as indicated in Table 1, with the resultant agreement for fair/poor scores between 3-5\%.

Table 3. The pBRA scores for four categorical scales

\begin{tabular}{|c|c|c|c|c|c|c|}
\hline & \multicolumn{4}{|c|}{ Median (range) } & \multicolumn{2}{|c|}{$\begin{array}{c}\text { Dichotomised scores normal/excellent/good } \\
\text { vs fair/poor }\end{array}$} \\
\hline & Excellent or normal & Good & Fair & Poor & Median & Wilcoxon rank-sum test, $P$ \\
\hline BCCT.core & $7.0(1-21)$ & $9.5(1-29)$ & $13.7(2-29)$ & $18.6(10-36)$ & 8.0 vs 14.8 & $<0.001$ \\
\hline Panel & $6.5(1-19)$ & $9.7(1-23)$ & $13.6(2-29)$ & $25.0(9-36)$ & 9.0 vs 14.5 & $<0.001$ \\
\hline Clinician & $8.4(1-31)$ & $10.9(1-29)$ & $11.9(1-26)$ & $23.1(9-36)$ & 9.2 vs 14.2 & 0.002 \\
\hline Patient & $8.4(1-29)$ & $10.7(1-36)$ & $13.4(4-31)$ & $13.7(7-17)$ & 9.4 vs 13.4 & 0.008 \\
\hline
\end{tabular}

vs, versus. 
The high level of agreement between BCCT.core and panel evaluation, and between patient and clinician direct assessments suggests that either method could potentially replace the other that it is concordant with which it is concordant. Between the former two methods, it is clear that panel evaluation is time consuming and could be impractical in centres with medium to large number of patients. It is also a subjective method making it less reproducible and is dependent on the experience of the panel individuals. ${ }^{13,14,29}$ Reliability and reproducibility of BCCT.core compared to panel evaluation was also demonstrated in a study by Heil et al. ${ }^{30}$ that assessed cosmesis using standardised photographs of 50 patients.

BCCT.core on the other hand, is objective and therefore reproducible, time efficient and easy to use. The results of this software analysis have been validated by an expert panel rating. ${ }^{22,23,29}$ And in a recent study, cosmetic outcome was assessed on a cohort of patients over time using BCCT.core. The mean BCCT.core scores in the study were shown to deteriorate from before surgery to the time point shortly after surgery to the long-term follow-up assessment reflecting the ability of the tool to measure the impact of the surgical interventions. ${ }^{31}$ Furthermore, when compared to BRA, the other objective assessment method, BCCT.core is more comprehensive as it includes other indices such as scar visibility and skin colour in addition to asymmetry, which is only what BRA assesses in a method restricted to relative nipple position. Although nipple position is an expression of changes in shape and volume of the breast and its asymmetry is the most striking visible parameter, but correlation between nipple position and cosmetic outcome may be less clear in cases where compromise in cosmetic outcome is mainly due to localised skin damage such as disturbing scars or severe telangiectasia, or in cases where breast contour deformity is localised without changes in nipple position, yet still affecting cosmesis significantly. ${ }^{16}$

With all the available objective methods however, the criticism for not taking into account the unmeasurable aspects of aesthetic outcome such as degree of fibrosis or breast oedema remains valid.

Patient's self-assessment is in no doubt an integral part of assessing cosmetic outcome but it is influenced by factors other than surgical techniques and radiotherapy only. Furthermore, the lack of its reproducibility, makes it inadequate to use for comparison of outcomes between centres, ${ }^{10,15,25,32-34}$ necessitating the need to improve and validate objective methods to allow for comparison of different surgical and radiation techniques with less confounding variables. ${ }^{35}$

Practical application to combine BCCT.core scores and patient reported cosmetic outcome needs further exploration. A suggested concept of this application would be based on the previously reported strong association between patient reported cosmetic outcome and Quality Of Life (QOL) and breast symptoms with QOL. ${ }^{36} \mathrm{~A}$ QOL adjusted cosmetic score, along the lines of QALY (Quality-Adjusted Life Year) or Q-TWiST (Quality-adjusted Time Without Symptoms or Toxicity), could then be derived by assigning utility to the BCCT.core score according to a factor derived from the patient reported cosmetic outcome or perhaps additional patient reported outcomes such as breast symptoms. Detailed modelling of this practical application is beyond the scope of this paper and would be explored in subsequent studies.

It is important to point out that in the population of our study, only $75 \%$ of patients consented to photography and in reality photographs could also be missed for logistical reasons such as forgetful clinical assessors or overburdened clinics. It is therefore recommended to consider all patients for photography by consenting them early on and introducing photography as a routine part of patients' assessments at baseline and the subsequent follow-up reviews. A reminder system for clinicians or other trained personnel to obtain photographs at certain time points is suggested. This would avoid blunting the usefulness of a photograph based objective evaluation method such as BCCT.core.

Recommendations for aesthetic evaluation previously published by Cardoso et al., have outlined the minimum standards required for photographs in relation to lighting, background, distance from camera and timing of evaluation among other details. ${ }^{11}$ We would suggest following the same recommendations to set the standards for a more homogenous and reproducible evaluation of cosmetic outcome.

In conclusion, based on the agreement scores between the different evaluation methods and by taking into account the individual advantages and disadvantages of each of these methods, we concluded that incorporating both BCCT.core assessment and patient self-assessment could potentially provide the basis of a gold standard method of breast cosmetic evaluation. BCCT.core represents an easy, time efficient, reproducible, cost-effective and reliable method, however, it lacks the functional and psychosocial elements of cosmesis that only patient selfreported outcomes can provide. A potential practical application combining scores from both assessment methods can be explored further in future studies.

\section{References}

1. Sarrazin D, Le MG, Arriagada $R$ et al. Ten-year results of a randomized trial comparing a conservative treatment to mastectomy in early breast cancer. Radiother Oncol 1989; 14: 177-84.

2. Blichert-Toft M, Rose C, Andersen JA et al. Danish randomized trial comparing breast conservation therapy with mastectomy: six years of life-table analysis. Danish Breast Cancer Cooperative Group. J Nat Cancer Instit Monographs 1992; 11: 19-25.

3. Veronesi U, Cascinelli N, Mariani L et al. Twenty-year follow-up of a randomized study comparing breast- 
conserving surgery with radical mastectomy for early breast cancer. New Engl J Med 2002; 347: 1227-32.

4. Fisher B, Anderson S, Bryant J et al. Twenty-year follow-up of a randomized trial comparing total mastectomy, lumpectomy, and lumpectomy plus irradiation for the treatment of invasive breast cancer. New Engl J Med 2002; 347: 1233-41.

5. van Dongen JA, Bartelink H, Fentiman IS et al. Randomized clinical trial to assess the value of breastconserving therapy in stage I and II breast cancer, EORTC 10801 trial. J Nat Cancer Instit Monographs 1992; 11: 15-8.

6. Munshi A, Kakkar S, Bhutani R, Jalali R, Budrukkar A, Dinshaw KA. Factors influencing cosmetic outcome in breast conservation. Clin Oncol (R Coll Radiol) 2009; 21: 285-93.

7. Group ST, Bentzen SM, Agrawal RK et al. The UK Standardisation of Breast Radiotherapy (START) Trial B of radiotherapy hypofractionation for treatment of early breast cancer: a randomised trial. Lancet 2008; 371: 1098-107.

8. Whelan TJ, Pignol JP, Levine MN et al. Long-term results of hypofractionated radiation therapy for breast cancer. New Engl J Med 2010; 362: 513-20.

9. Mukesh MB, Barnett GC, Wilkinson JS et al. Randomized controlled trial of intensity-modulated radiotherapy for early breast cancer: 5-year results confirm superior overall cosmesis. J Clin Oncol 2013; 31: 4488-95.

10. Christie DRH, O'Brien MY, Christie JA et al. A comparison of methods of cosmetic assessment in breast conservation treatment. Breast 1996; 5: 358-67.

11. Cardoso MJ, Cardoso JS, Vrieling C et al. Recommendations for the aesthetic evaluation of breast cancer conservative treatment. Breast Cancer Res Treat 2012; 135: 629-37.

12. Haloua $\mathrm{MH}$, Krekel NM, Jacobs GJ et al. Cosmetic outcome assessment following breast-conserving therapy: a comparison between BCCT.core Software and Panel Evaluation. Int. J Breast Cancer 2014; 2014: 716860.

13. Harris JR, Levene MB, Svensson G, Hellman S. Analysis of cosmetic results following primary radiation therapy for stages I and II carcinoma of the breast. Int J Radiat Oncol Biol Phys 1979; 5: 257-61.

14. Pezner RD, Lipsett JA, Vora NL, Desai KR. Limited usefulness of observer-based cosmesis scales employed to evaluate patients treated conservatively for breast cancer. Int J Radiat Oncol Biol Phys 1985; 11: 1117-9.

15. Vrieling C, Collette L, Bartelink E et al. Validation of the methods of cosmetic assessment after breastconserving therapy in the EORTC "boost versus no boost" trial. EORTC Radiotherapy and Breast Cancer Cooperative Groups. European Organization for Research and Treatment of Cancer. Int J Radiat Oncol Biol Phys 1999; 45: 667-76.
16. Van Limbergen E, van der Schueren EVan Tongelen K. Cosmetic evaluation of breast conserving treatment for mammary cancer. 1. Proposal of a quantitative scoring system. Radiother Oncol 1989; 16: 159-67.

17. Tsouskas LI, Fentiman IS. Breast compliance: a new method for evaluation of cosmetic outcome after conservative treatment of early breast cancer. Breast Cancer Res Treat 1990; 15: 185-90.

18. Noguchi M, Saito Y, Mizukami Y et al. Breast deformity, its correction, and assessment of breast conserving surgery. Breast Cancer Res Treat 1991; 18: 111-8.

19. Al-Ghazal SK, Fallowfield L, Blamey RW. Patient evaluation of cosmetic outcome after conserving surgery for treatment of primary breast cancer. Eur J Surg Oncol 1999; 25: 344-6.

20. Hau E, Browne LH, Khanna S et al. Radiotherapy breast boost with reduced wholebreast dose is associated with improved cosmesis: the results of a comprehensive assessment from the St. George and Wollongong randomized breast boost trial. Int J Radiat Oncol Biol Phys 2012; 82: 682-9.

21. Pezner RD, Patterson MP, Hill LR et al. Breast retraction assessment: an objective evaluation of cosmetic results of patients treated conservatively for breast cancer. Int J Radiat Oncol Biol Phys 1985; 11: 575-8.

22. Cardoso JS, Cardoso MJ. Towards an intelligent medical system for the aesthetic evaluation of breast cancer conservative treatment. Artif Intell Med 2007; 40: 115-26.

23. Cardoso MJ, Cardoso J, Amaral $\mathrm{N}$ et al. Turning subjective into objective: the BCCT.core software for evaluation of cosmetic results in breast cancer conservative treatment. Breast 2007; 16: 456-61.

24. Hoeller U, Kuhlmey A, Bajrovic A et al. Cosmesis from the patient's and the doctor's view. Int J Radiat Oncol Biol Phys 2003; 57: 345-54.

25. Borger JH, Keijser AH. Conservative breast cancer treatment: analysis of cosmetic results and the role of concomitant adjuvant chemotherapy. Int J Radiat Oncol Biol Phys 1987; 13: 1173-7.

26. Sneeuw KC, Aaronson NK, Yarnold JR et al. Cosmetic and functional outcomes of breast conserving treatment for early stage breast cancer. 1 . Comparison of patients' ratings, observers' ratings, and objective assessments. Radiother Oncol 1992; 25: 153-9.

27. Vass S, Bairati I. A cosmetic evaluation of breast cancer treatment: a randomized study of radiotherapy boost technique. Int J Radiat Oncol Biol Phys 2005; 62: 1274-82.

28. Byrt T, Bishop J, Carlin JB. Bias, prevalence and kappa. J Clin Epidemiol 1993; 46: 423-9.

29. Cardoso MJ, Cardoso J, Santos AC, Barros H, Cardoso de Oliveira M. Interobserver agreement and consensus over the esthetic evaluation of conservative treatment for breast cancer. Breast 2006; 15: 52-7.

30. Heil J, Carolus A, Dahlkamp J et al. Objective assessment of aesthetic outcome after breast conserving therapy: subjective third party panel rating 
and objective BCCT.core software evaluation. Breast 2012; 21: 61-5.

31. Hennigs A, Hartmann B, Rauch G et al. Long-term objective esthetic outcome after breast-conserving therapy. Breast Cancer Res Treat 2015; 153: 345-51.

32. Kaija H, Rauni S, Jorma I, Matti H. Consistency of and doctor-assessed cosmetic outcome after conservative treatment of breast cancer. Breast Cancer Res Treat 1997; 45: 225-8.

33. Liljegren G, Holmberg L, Westman G. The cosmetic outcome in early breast cancer treated with sector resection with or without radiotherapy. Uppsala-Orebro Breast Cancer Study Group. Eur J Cancer 1993; 29A: 2083-9.
34. Sacchini V, Luini A, Agresti R et al. The influence of radiotherapy on cosmetic outcome after breast conservative surgery. Int J Radiat Oncol Biol Phys 1995; 33: 59-64.

35. Heil J, Dahlkamp J, Golatta M et al. Aesthetics in breast conserving therapy: do objectively measured results match patients' evaluations? Ann Surg Oncol 2011; 18: 134-8.

36. Hau E, Browne L, Capp A et al. The impact of breast cosmetic and functional outcomes on quality of life: long-term results from the St. George and Wollongong randomized breast boost trial. Breast Cancer Res Treat 2013; 139: 115-23. 